\title{
Enhanced Anti-Brain Metastasis from Non-Small Cell Lung Cancer of Osimertinib and Doxorubicin Co-Delivery Targeted Nanocarrier
}

This article was published in the following Dove Press journal: International Journal of Nanomedicine

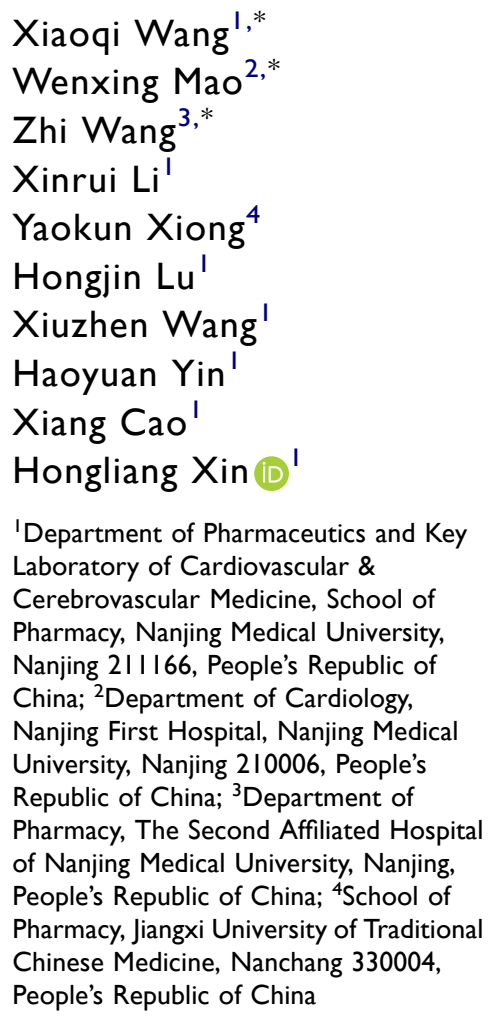

*These authors contributed equally to this work

\begin{abstract}
Purpose: Currently, the treatment of brain metastases from non-small cell lung cancer (NSCLC) is rather difficult in the clinic. A combination of small molecule-targeted drug and chemo-drug is a promising therapeutic strategy for the treatment of NSCLC brain metastases. But the efficacy of this combination therapy is not satisfactory due to the blood-brain barrier (BBB). Therefore, it is urgent to develop a drug delivery system to enhance the synergistic therapeutic effects of small molecule-targeted drug and chemo-drug for the treatment of NSCLC brain metastases.
\end{abstract}

Methods: T7 peptide installed and osimertinib (AZD9291) loaded intracellular glutathione (GSH) responsive doxorubicin prodrug self-assembly nanocarriers (T7-DSNPs/9291) have been developed as a targeted co-delivery system to enhance the combined therapeutic effect on brain metastases from NSCLC. In vitro cell experiments, including intracellular uptake assay, in vitro BBB transportation, and MTT assay were used to demonstrate the efficacy of T7-DSNPs/9291 in NSCLC brain metastasis in vitro. Real-time fluorescence imaging analysis, magnetic resonance imaging analysis, and Kaplan-Meier survival curves were used to study the effect of T7-DSNPs/9291 on an animal model in vivo.

Results: T7-DSNPs/9291 could significantly enhance BBB penetration of AZD9291 and doxorubicin via transferrin receptor-mediated transcytosis. Moreover, T7-DSNPs/9291 showed significant anti-NSCLC brain metastasis effect and prolonged median survival of an intracranial NSCLC brain metastasis animal model.

Conclusion: T7-DSNPs/9291 is a potential drug delivery system for the combined therapy of brain metastasis from NSCLC.

Keywords: drug delivery, brain metastasis, AZD9291, redox-responsive, brain targeted delivery system

\section{Introduction}

Lung cancer is the most serious cause of cancer death in humans. ${ }^{1}$ About 1/3 of non-small cell lung cancer (NSCLC) patients have brain metastasis during the follow-up standard-of-care treatment. ${ }^{2}$ Although symptomatic treatments such as the application of dehydration drugs and anti-tumor treatments such as whole-brain radiotherapy have brought some benefits to patients, the therapy of brain metastases from NSCLC is rather difficult, and the prognosis is still poor. ${ }^{3-6}$

Epidermal growth factor receptor-tyrosine kinase inhibitor (EGFR-TKI) has been adopted in the clinical treatment of NSCLC. Osimertinib (AZD9291), the third-generation EGFR-TKI, is a mutant selective irreversible EGFR inhibitor that
Correspondence: Hongliang Xin Fax +86 $2586868467 ;+862586868477$ Email xhl@njmu.edu.cn
International Journal of Nanomedicine 2020:I5 549I-550I 
is effective against both EGFR-TKI-sensitive mutations and T790M-resistant mutations. ${ }^{7}$ It is reported that AZD9291 showed better potential for the treatment of the brain metastasis from NSCLC and a lessened risk of brain progression compared with other EGFR-TKIs. ${ }^{8-10}$ However, the permeability of EGFR-TKIs into the brain is very limited because of the blood-brain barrier (BBB) and drug efflux proteins such as P-glycoprotein and breast cancer resistance protein which are overexpressed along with the BBB. ${ }^{11-13}$ Therefore, it is urgent to enhance the brain permeability of AZD9291 to improve its therapeutic effect against brain metastasis from NSCLC.

A combination of different classes of drugs has been widely used in cancer therapy, which has overcome the common issues of drug resistance and toxicity caused by monotherapy, and achieved a better curative effect. ${ }^{14}$ It is reported that AZD9291 combined with chemotherapeutic drugs is a potential therapeutic schedule of brain metastasis from NSCLC. ${ }^{15}$ Doxorubicin (DOX) is one of the original anthracyclines leading to DNA damage and cell death, ${ }^{16}$ which has been used in combination therapy for NSCLC. ${ }^{17}$ However, due to the different rates of metabolism within the body, the different properties of the pharmacokinetics and biodistribution of multiple drugs can lead to constant changes in the ratio of drugs, preventing drugs from being effective. ${ }^{18}$ Therefore, a co-delivery system has been developed to avoid this limitation by carrying multiple therapeutic agents in a nanocarrier which can bring the drug to the tumor site. ${ }^{19-21}$ In addition, it is necessary to construct a delivery system for stimulatory response in tumor cells, which enables the triggered release of AZD9291 and DOX in the NSCLC brain metastasis tumor cells. Studies have shown that the intracellular glutathione (GSH) concentration is 1000 times higher than extracellular levels, and this has become an important "switching molecule" that controls the intracellular intelligent release of drugs. ${ }^{22-24}$ The disulfide bond (-S-S-) is the most commonly used functionalized linker for the development of an intracellular GSH-responsive drug delivery system, ${ }^{25,26}$ which has been demonstrated to be stable in the blood circulation system but can split rapidly in tumor cells triggered by the reductive microenvironment. ${ }^{27-29}$ Thus, in this study, the GSHresponsive DOX prodrug (DOX-S-S-C18) conjugate was used as the nanocarrier backbone, as it can form nanoparticles without any surfactant with an ultra-high AZD9291 loading content. Furthermore, in order to improve the BBB penetration, the nanocarrier was installed with a BBB targeting moiety (T7 peptide), which was mediated transcytosis by transferrin receptor (TfR).

Herein, T7-installed AZD9291-loaded PEGylated DOX-SS- $\mathrm{C}_{18}$ conjugate self-assembled nanocarrier (T7DSNPs/9291) was constructed (Figure 1). After intravenous (i.v.) injection, T7- DSNPs/9291 was expected to be stable in the physiological condition to avoid drug premature release and reduce the toxicity of the offtarget effect. Then, T7 peptide could enhance the penetration of T7-DSNPs/9291 across BBB through transferrin-mediated transcytosis. When the T7-DSNPs /9291 was internalized into the NSCLC brain metastasis tumor cells, the nanocarrier could be disassembled under high intracellular GSH level and stimuli-responsively released AZD9291 and DOX which acted on the respective target to enhance the anti-brain metastasis from NSCLC (Figure 1).

\section{Experimental}

Materials

Doxorubicin and AZD9291 were purchased from Hubei Dixin Chemical Manufacturing Co., Ltd. (Wuhan, China). T7 (CHAIYPRH) peptide was obtained by GL Biochem Co., Ltd. (Shanghai, China). T7-PEG-DSPE was synthesized in line with our previous report. ${ }^{30,31} \mathrm{MeO}-\mathrm{PEG} 2000$ DSPE and Maleimide-PEG2000-DSPE were obtained from CordenPharma Co., Switzerland. All the other reagents were used as received.

\section{Animals and Cell Lines}

Male Balb/c nude mice weighing $20 \pm 2 \mathrm{~g}$ were obtained from BK animal Ltd. (Shanghai, China). PC-9 cells (human lung cancer cells) and BCEC cells (rat's brain capillary endothelial cell line) were purchased from the Institute of Biochemistry and Cell Biology, Shanghai Institutes for Biological Sciences, Chinese Academy of Sciences (Shanghai, China).

\section{Preparation of Nanocarrier}

The DOX-SS- $\mathrm{C}_{18}$ conjugate was synthesized as provided in the Synthesis of GSH-responsive DOX prodrug (DOX-SSC18) section of the Supporting Information. The organic solvent evaporation was employed to prepare DOX-SS- $\mathrm{C}_{18}$ conjugate self-assembled nanoparticles (DSNPs). Briefly, DOX-SS- $\mathrm{C}_{18}$ conjugates $(10 \mathrm{mg})$ were dissolved in tetrahydrofuran $(200 \mu \mathrm{L})$ first. The resulting solution was then 


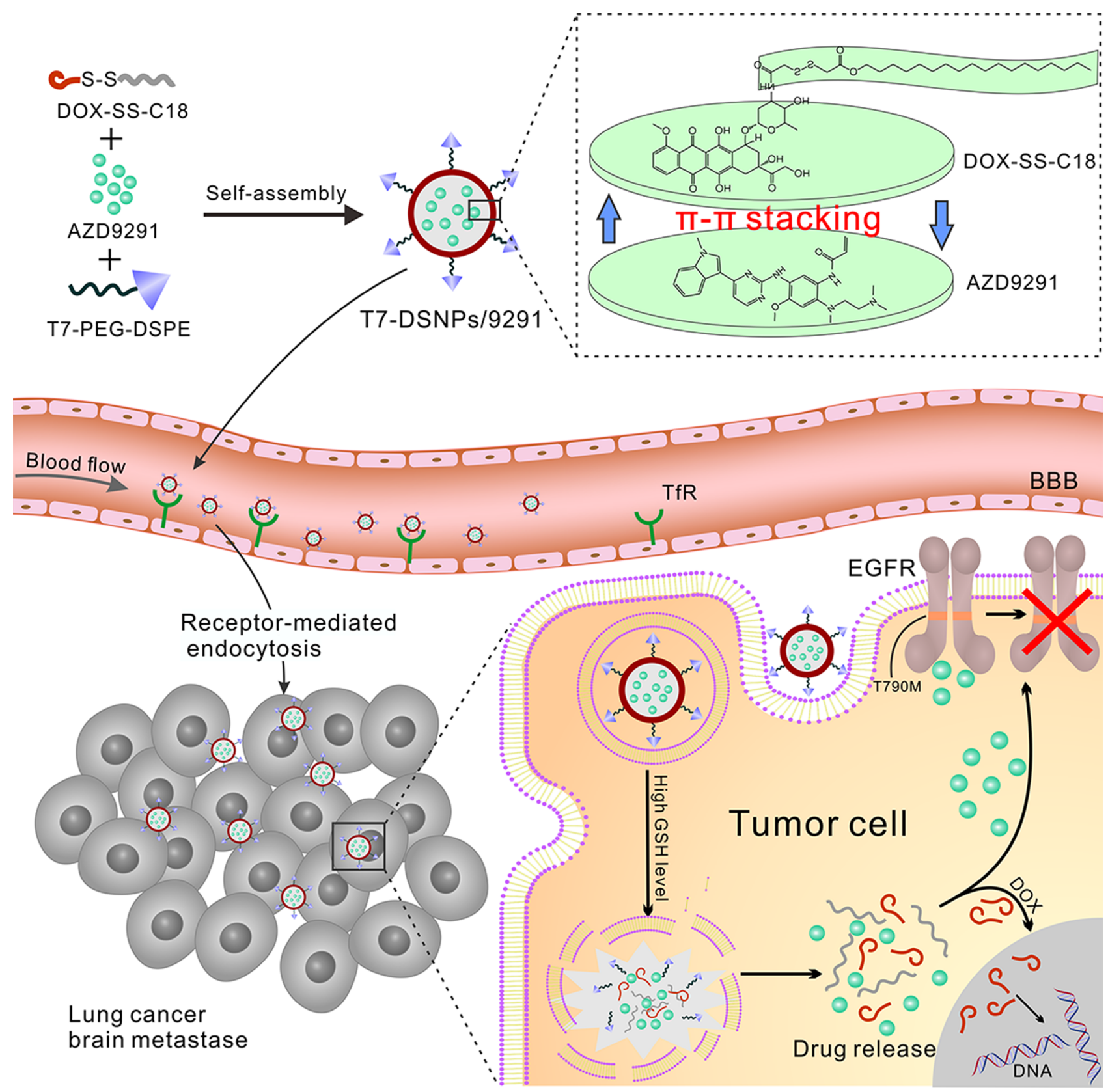

Figure I Schematic design of AZD929I-loaded T7 modified PEGylated DOX-SS-C 18 conjugated self-assembled nanocarrier (T7-DSNPs/929I) for the treatment of brain metastases from NSCLC.

injected dropwise into distilled water $(20 \mathrm{~mL})$. After stirring for 5 minutes, the organic solvent in the solution was further eliminated by rotary evaporator, and the $0.22 \mu \mathrm{m}$ mixed cellulose (MCE) syringe filter was used to filter the nanoparticles. AZD9291-loaded DSNPs (DSNPs/9291) were prepared by the above method with AZD9291 (5 mg) dissolved in an organic solvent. AZD9291-loaded PEGylated DOX-SS-C ${ }_{18}$ conjugate nanoparticles (PEGDSNPs/9291) and T7-DSNPs/9291 were prepared by adding MeO-PEG2000-DSPE or T7-PEG-DSPE (T7-PEG-DSPE
/DSNPs $=15 / 100, \mathrm{w} / \mathrm{w} ;$ MeO-PEG-DSPE or T7-PEGDSPE/DSNPs $/ 9291=10 / 100, \mathrm{w} / \mathrm{w}$ ) in an organic solvent. Coumarin-6 or Dir fluorescence-labeled nanoparticles were prepared via coumarin-6 or Dir co-dissolved with AZD9291 in the organic solvent during nanoparticle preparation.

\section{Physicochemical Characterization of Nanocarrier}

Morphology of the nanocarrier was observed by transmission electronic microscopy (TEM) (Tecnai G2 Spirit Bio 
TWIN, FEI, USA). The $\zeta$ potential, particle size, and size distribution of the nanocarrier were measured by DLS (Zs90, Malvern, UK). The experimental method for disassembled nanoparticles was shown in the Reduction-triggered disassembly of T7-DSNPs/9291 section.

\section{In vitro Release}

The in vitro release behavior of T7-DSNPs/9291 was investigated by ultrafiltration centrifugation. The release media was buffer solution containing $1 \mu \mathrm{M} \mathrm{GSH}$ and $10 \mathrm{mM} \mathrm{GSH}$, respectively. Briefly, T7-DSNPs/9291 was scattered into $5 \mathrm{~mL}$ of release medium and shaken at $37^{\circ} \mathrm{C}$ at the speed of $150 \mathrm{rpm} / \mathrm{min}$. Then, the solution was transferred to an ultrafiltration device ( $\mathrm{MWCO}=30 \mathrm{~K} \mathrm{Da})$ at the predetermined time. After centrifuged at $4500 \mathrm{rpm}$, the DOX and AZD9291 concentration of filtrate were measured by high performance liquid chromatography.

\section{In vitro Uptake Evaluation}

For the qualitative experiment, BCEC cells $\left(2 \times 10^{4}\right.$ cells/ well) were seeded into 24-well plates. After $24 \mathrm{~h}$, the cells were administrated by various coumarin- 6 fluorescencelabeled nanocarriers at 1,2 , and $5 \mu \mathrm{g} / \mathrm{mL}$, respectively. After washing by pre-cooled PBS buffer 3 times, the cells were fixed in $4 \%(\mathrm{w} / \mathrm{v})$ paraformaldehyde solution at room temperature. Finally, the cells were stained by Hoechst 33,342 and then viewed by fluorescent microscopy (Imager A1, Zeiss, Germany). The qualitative analysis on PC-9 cells was shown in the Qualitative analysis of PC-9 cells uptake of T7-DSNPs/9291 section of the Supporting Information.

For quantitative evaluation, BCEC cells $\left(1 \times 10^{5}\right.$ cells/ well) were seeded into 24 -well plates. The cell culture and administration methods were the same as those referred to in the qualitative experiment. Then, the cells were lysed by $1 \%$ TritonX-100. The cell protein content was measured by the BCA protein assay. The coumarin- 6 concentration of the lysate was detected by a fluorescence spectrophotometer.

\section{In vitro $B B B$ Transportation}

The BBB model was constructed as previously. ${ }^{32}$ BCEC $\left(1 \times 10^{4}\right.$ cells/pore) was seeded on a transwell filter. When $90 \%$ confluence was reached, the cells were serum-starved with $1 \mu \mathrm{mol} / \mathrm{L}$ hydrocortisone for another 3 days. After monitoring transepithelial electrical resistance (TEER), the cells were used for BBB transportation evaluation. Free AZD9291 + DOX, PEG-DSNPs/9291, and T7-DSNPs 19291 (equivalent to $10 \mu \mathrm{g} / \mathrm{mL}$ AZD9291) were dropped into the top chamber. After incubation for $2 \mathrm{~h}, 800 \mu \mathrm{L}$ samples were taken out from the base chamber. For the ligand competition inhibition evaluation, T7 $(50 \mu \mathrm{g} / \mathrm{mL})$ was administrated into the top chamber beforehand. After $1 \mathrm{~h}$ incubation, the T7 solution was substituted by the T7DSNPs/9291 solution, and the next steps were consistent with the above-mentioned steps. The DOX and AZD9291 concentration in the base chamber was detected by high performance liquid chromatography.

\section{In vitro Cytotoxicity Assay}

The in vitro cytotoxicity of T7-DSNPs/9291 was evaluated by MTT assay. Briefly, BCEC or PC-9 cells $\left(4 \times 10^{3}\right.$ cells/well) were seeded into 96-well plates. Twenty-four hours later, the medium was substituted by various formulations at the different concentrations of AZD9291. After $48 \mathrm{~h}$ incubation, the MTT solution was added into the well. Four hours later, $200 \mu \mathrm{L}$ DMSO was added into each well to dissolve the crystal. The absorbance was measured by a microplate reader at $490 \mathrm{~nm}$ (Thermo Multiskan MK3, USA).

\section{In vivo Real-Time Fluorescence Imaging}

An intracranial PC-9 tumor mice model was constructed as reported. ${ }^{33}$ Briefly, $4 \mu \mathrm{L}$ PC-9 cell suspension $\left(2 \times 10^{5}\right.$ cells) was implanted into the right striatum of anesthetized $\mathrm{BAlB} / \mathrm{c}$ nude mice by chloral hydrate at a rate of 0.8 $\mu \mathrm{L} / \mathrm{min}$. After 21 days, these mice were then randomized for subsequent studies. Intracranial PC-9 tumor mice were administrated with $250 \mu \mathrm{L}$ Dir labeled T7-DSNPs/9291 and PEG-DSNPs/9291 via tail vein and imaged using the in vivo imaging system (IVIS Spectrum, PerkinElmer, USA) at 4,12 , and $24 \mathrm{~h}$ post-administration, respectively. To investigate the distribution of various nanoparticles in the tumor site and primary organs, these mice were executed and the primary organs were harvested and imaged by IVIS Spectrum after $24 \mathrm{~h}$. After fixing in $4 \%$ paraformaldehyde and dehydrating in $15 \%$ and $30 \%$ sucrose solution, respectively, the brain tissues were cut by the frozen microtome. Afterward, the brain sections were stained with DAPI for $10 \mathrm{~min}$. The distribution of nanoparticles in the brain section was viewed by a laser confocal microscope.

\section{In vivo Anti-Tumor Efficacy}

The in vivo anti-brain metastasis from NSCL of T7-DSNPs /9291 was studied by established intracranial PC- 9 tumor mice. Each group contained 8 mice were injected with saline, DOX +AZD9291, T7-DSNPs, PEG-DSNPs/9291, and T7-DSNPs/ 
9291 at a dose of $5 \mathrm{mg} / \mathrm{kg}$ AZD9291 and $6 \mathrm{mg} / \mathrm{kg}$ DOX, respectively, on 2, 4, 6, and 8 days after PC-9 transplantation. On day 21, the intracranial tumor growth of the mice was monitored by magnetic resonance imaging (MRI). KaplanMeier survival curves of each group mice were also plotted.

\section{Statistical Analysis}

These data were statistically analyzed by one-way ANOVA and two-way ANOVA using SPSS 22.0. Asterisks are used to indicate statistical significance $\left({ }^{*} P<0.05\right.$, ${ }^{*} P<0.01$, $* * * P<0.001)$.

\section{Results and Discussion Characterization of T7-DSNPs/929I}

The structure of DOX-SS-C ${ }_{18}$ conjugate was characterized by $\mathrm{MS},{ }^{1} \mathrm{H} \mathrm{NMR}$, and ${ }^{13} \mathrm{C}$ NMR (Supporting information, Figure $\mathrm{S} 1)$. The MS (m/z) of DOX-SS- $\mathrm{C}_{18}$ : calculated for $\left[\mathrm{C}_{49} \mathrm{H}_{69} \mathrm{NO}_{14} \mathrm{~S}_{2}\right][\mathrm{M}]+=959$, found: 958.93 . The ${ }^{1} \mathrm{H} \mathrm{NMR}$ spectra of DOX-SS-C ${ }_{18}(\mathrm{DMSO}, \delta): 13.98(\mathrm{~s}, 1 \mathrm{H}, 11-\mathrm{OH})$, 13.21(s, 1H, 6-OH), 7.88-7.55(m, 4H, 2-H, 3-H, 4-H, $\left.4^{\prime}-\mathrm{NH}\right), 5.44\left(\mathrm{~s}, 1 \mathrm{H}, 2^{\prime}-\mathrm{H}\right), 5.23\left(\mathrm{~d}, 1 \mathrm{H}, 5^{\prime}-\mathrm{H}\right), 4.88-4.85$ $(\mathrm{m}, 3 \mathrm{H}, 14-2 \mathrm{H}, \mathrm{OH}), 4.60-4.58\left(\mathrm{~d}, 2 \mathrm{H}, 4^{\prime}-\mathrm{H}, 10-\mathrm{H}\right)$, $4.18-4.16(\mathrm{~m}, 1 \mathrm{H}, \mathrm{OH}), 4.02-3.94(\mathrm{~m}, 6 \mathrm{H}, 15-3 \mathrm{H}, 5$ "-2H, $\mathrm{OH}), \quad 3.67(\mathrm{~s}, \quad 2 \mathrm{H}, 22-" \mathrm{H}), 3.48-3.46(\mathrm{~m}, 3 \mathrm{H}, 32-" \mathrm{H}$, $\left.6^{\prime}-\mathrm{H}\right), 2.93-2.82(\mathrm{dd}, 2 \mathrm{H}, 7-2 \mathrm{H}), 2.19-2.10\left(\mathrm{~m}, 2 \mathrm{H}, 3^{\prime}-\right.$ 2H), 1.86-1.13(m, 37H, 9-2H, 7'-3H, 632-"21 "H), 0.86-$0.82(\mathrm{t}, 3 \mathrm{H}, 223-" \mathrm{H})$. The ${ }^{13} \mathrm{C}$ NMR spectra of DOX-SS $-\mathrm{C}^{18}$ (DMSO, $\left.\delta\right): 214.38(\mathrm{C} 13), 186.77-186.65(\mathrm{C} 12, \mathrm{C} 5)$, $169.58(\mathrm{C} 1167.37,(")(\mathrm{C} 4161.16,(\mathrm{C} 1), \quad 156.58(\mathrm{C} 6)$, 154.97(C11), 136.58-134.48(C3, C10a, C4a, C6a), 120.24-119.34(C12a, C2, C4), 111.06-110.94(C11a, C5a), 100.83(C2'), 75.38(C8), 70.40-67.10(C6', C5', C10), 65.24(C564.21, ,("C14), 56.96(C15), 45.88(C4'), 42.22 (C236.98-37.02, ,(C3', C325.71-31.79, ,(C6"20-", C9, C7), 22.59(C2117.46, ,("C7'), 14.22(C22"). These results showed that the DOX-SS-C 18 conjugate was synthesized successfully.

In this study, T7-DSNPS/9291 was prepared via organic solvent evaporation. The driving forces for the self-assembly of the nanocarrier may be non-bonded hydrophobic interactions, and the balance of the intermolecular force and the establishment of a favorable conformation may indicate that $\mathrm{S}-\mathrm{S}$ bonds show a distinct preference for dihedral angles approaching $90^{\circ} .^{25}$ Furthermore, the T7 peptide was modified on the surface of the nanoparticles due to electrostatic adsorption, or it was engaged in NH- $\pi$ stacking with doxorubicin, in which the amide NH of T7 interacts with $\pi$ cloud of doxorubicin. $^{34}$ The properties of various nanoparticles including $\zeta$ potential, particle size, and drug loading content are shown in Supporting information, Table S1. The drug loading content of AZD9291 and DOX reached 28.62\% and $34.34 \%$, respectively. The ultra-high drug loading of AZD9291 may result from the stable $\pi-\pi$ stacking between the phenyl group of the AZD9291 structure with the anthracene ring of the DOX structure or NH of T7 interaction with $\pi$ cloud of DOX. The particle size of DSNPS/9291, PEGDSNPS/9291, and T7-DSNPS/9291 was $85.19 \pm 0.85 \mathrm{~nm}$, $88.53 \pm 1.87 \mathrm{~nm}$, and $90.56 \pm 1.63 \mathrm{~nm}$, respectively. The PDI of all the nanoparticles performed decently. Meanwhile, TEM images showed uniformly spherical morphology of T7DSNPs/9291 (Figure 2A) and the size of the nanoparticles was around $100 \mathrm{~nm}$, which was consistent with the result of DLS (Figure 2B).

The TEM image of T7-DSNPs/9291 incubated with GSH (Figure 3A) and the size distribution of T7-DSNPs /9291 incubated with GSH during $8 \mathrm{~h}$ (Figure 3B) displayed the deformation and expansion of nanocarrier, which revealed T7-DSNPs/9291 had the GSH responsiveness. In the investigation of the triggered-release profile of T7DSNPs/9291 (Figure 3C), almost no AZD9291 and DOX were released under the presence of $1 \mu \mathrm{M}$ GSH. This phenomenon can be explained that the concentration of GSH is not high enough to disrupt the disulfide bond of T7-DSNPs /9291 in this condition, and the $\pi-\pi$ stacking between the phenyl group of the AZD9291 structure with the anthracene ring of the DOX structure is stable enough. In contrast, with the $10 \mathrm{mM}$ GSH incubation, sudden explosive release of AZD9291 and DOX was performed in the first hour, and the cumulative release of AZD9291 and DOX from T7-DSNPs /9291 reached $63.95 \%$ and $62.64 \%$ after 8 hours, respectively. These results indicated that T7-DSNPs/9291 might be stable in the circulation system to minimize the premature release of AZD9291 and DOX. Meanwhile, AZD9291 and
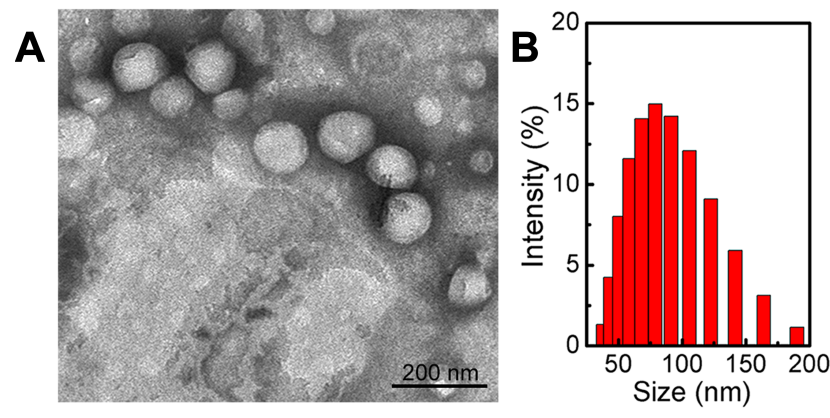

Figure 2 Characterization of T7-DSNPs/929I. TEM images of T7-DSNPs/929I(A) and DLS analysis of T7-DSNPs/9291 (B). 

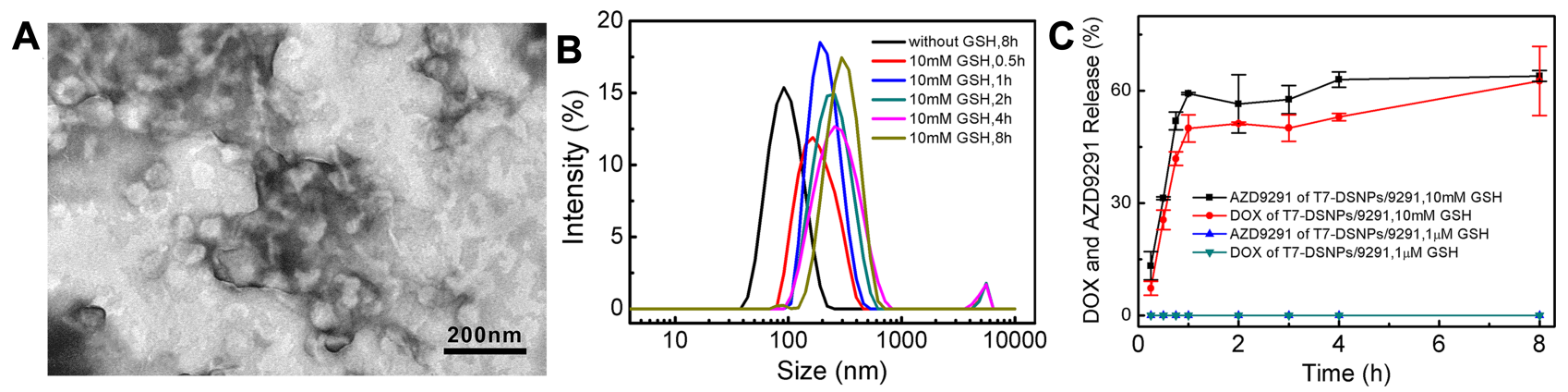

Figure 3 Response of nanoparticles to reductive stimulation. TEM images of T7-DSNPs/929I incubated with $10 \mathrm{mM} \mathrm{GSH} \mathrm{(A).} \mathrm{Size} \mathrm{distribution} \mathrm{of} \mathrm{T7-DSNPs/929I} \mathrm{in}$ response to GSH (B). Reduction-triggered release of payload from T7-DSNPs/929I in different release condition $(n=3)(\mathbf{C})$.

DOX could be rapidly released from the nanocarrier to inhibit tumor cells under the trigger of GSH when they were internalized into the cytoplasm. Therefore, the codelivery system developed in this study may minimize the premature release of therapeutic drugs and enhance the antitumor efficacy of AZD9291 and DOX.

\section{Cellular Uptake}

Coumarin-6 was used as a fluorescent probe to study the cellular uptake characteristics of T7-DSNPs/9291 qualitatively. The fluorescence of the T7-DSNPs/9291 group was significantly stronger than that of the PEG-DSNPs/9291 group with $1 \mathrm{~h}$ incubation at various concentrations of fluorescent probe (Figure 4A). In addition, the fluorescence of T7-DSNPs/9291 incubated for $2 \mathrm{~h}$ was significantly higher than that of the nanoparticles incubated for $1 \mathrm{~h}$. These results showed that uptake of nanoparticles by BCEC cells exhibited concentration- and time-dependently under various conditions, and the same conclusion was obtained in PC-9 cells (Supporting information, Figure S2). Quantitative cellular uptake of T7-DSNPs/9291 and PEG-DSNPs/9291 in BCEC cells also showed a concentration-dependent effect
(Figure 4B). According to these results, when the concentration of coumarin- 6 was $1,2,5$, and $10 \mu \mathrm{g} / \mathrm{mL}$, the cellular uptake of the T7-DSNPs/9291 was 1.86, 1.76, 1.54, and 1.62 times higher than the PEG-DSNPs/9291, respectively. Qualitative and quantitative analysis showed that T7 peptide modification can significantly increase the endocytosis of nanoparticles. Therefore, T7-DSNPs/9291 may be a potential nanocarrier for overcoming BBB.

\section{In vitro BBB Transportation}

To study the in vitro permeability across $\mathrm{BBB}$ of various formulations, free AZD9291 and DOX, PEG-DSNPs/9291, T7-DSNPs/9291 were put in the top chamber and the concentration of AZD9291 and DOX in the base chamber was monitored, respectively. From Figure $4 \mathrm{C}$, it is indicated that AZD9291 proportion of the free AZD9291+DOX, PEGDSNPs/9291, T7-DSNPs/9291, and T7 competitive inhibition of T7-DSNPs/9291 group across the BCEC monolayer was about $0.71 \%, 1.53 \%, 2.55 \%$, and $1.45 \%$, respectively, and the DOX proportion of the four groups was about $1.53 \%, 2.09 \%, 2.63 \%$, and $1.89 \%$, respectively, which indicated no significant difference in the transmittance of
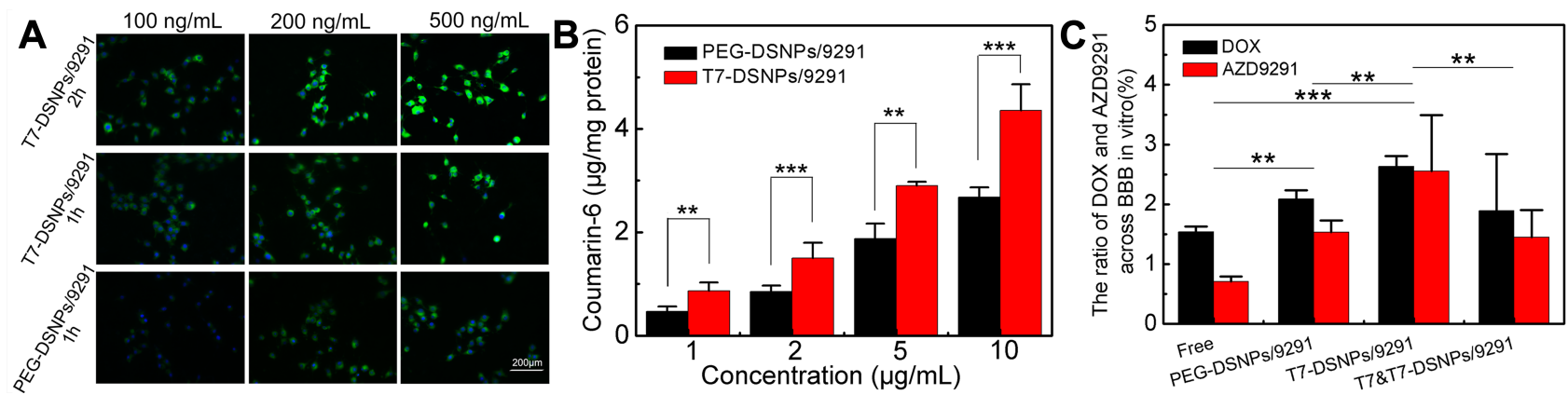

Figure 4 In vitro cellular uptake and penetration capacity of T7-DSNPs/929I. BCEC uptake of T7-DSNPs/929I and PEG-DSNPs/929I at $37^{\circ} \mathrm{C}$ incubated with I00, 200, and $500 \mathrm{ng} / \mathrm{mL}$ coumarin-6 for I h and $2 \mathrm{~h}$, respectively. Bar: $200 \mu \mathrm{m}(\mathbf{A})$. BCEC uptake of PEG-DSNPs/929I and T7-DSNPs/929I incubated with coumarin-6 concentrations ranged from I to $10 \mu \mathrm{g} / \mathrm{mL}(\mathrm{n}=3)(B)$. Trans-BBB transport of AZD929I and DOX, T7-DSNPs, PEG-DSNPs/929I, and T7-DSNPs/929I through in vitro BBB model ( $\mathrm{n}=3$ ) (C). ${ }^{*} * \mathrm{P}<0.00 \mathrm{I}$, $* * \mathrm{P}<0.0 \mathrm{l}$. 
AZD9291 and DOX of the same formulation. These results suggested the nanoparticle formulation could enhance AZD9291 and DOX transport across BCEC monolayer in vitro compared with that of free drug. Furthermore, the T7-modified group significantly increased BCEC monolayer penetration compared with the plain group. The transportation across BCEC monolayer of T7-DSNPs/9291 was higher than that of the one pre-incubated with $\mathrm{T} 7$ peptide, indicating that the transcytosis of T7-DSNPs/9291 across the BCEC monolayer is mediated by TfR, and T7-modified nanoparticles could increase the penetration of AZD9291 and DOX into the brain. Taken together, T7-DSNPs/9291 may improve the BBB permeability of AZD9291 and DOX, which is a benefit to the treatment of brain metastases from NSCLC.

\section{In vitro Cytotoxicity}

To evaluate the effect of T7-DSNPs/9291 on the BBB, the in vitro cytotoxicity of each formulation to BCEC cells was examined by MTT assay. As exhibited in Figure 5A, T7-DSNPs, PEG-DSNPs/9291, and T7-DSNPs/9291 had negligible toxicity to BCEC cells, ranging from 0.01 to 5 $\mu \mathrm{g} / \mathrm{mL}$ of AZD9291 and 0.012 to $6 \mu \mathrm{g} / \mathrm{mL}$ of DOX, probably owing to the insufficient GSH inside of BCEC, resulting in disulfide bonds not being disrupted to release AZD9291 and DOX. Meanwhile, free AZD9291 and DOX significantly inhibited the growth of BCEC cells at the same concentration. These results indicated that the codelivery nanocarrier could relieve the toxicity of AZD9291 and DOX to the blood-brain barrier.

However, at the same dose of AZD9291 and DOX, it was shown that the cytotoxicity of various AZD9291 formulations to PC-9 cells was concentration-dependent, and the cytotoxicity of T7-DSNPs/9291 (IC50 value of
AZD9291 and DOX: $2.041 \pm 0.31 \mu \mathrm{g} / \mathrm{mL}$ and $2.449 \pm 0.389$ $\mu \mathrm{g} / \mathrm{mL}$ ) was evidently higher compared with the T7-DSNPs group $\left(\mathrm{IC}_{50}\right.$ value of $\left.\mathrm{DOX}: 11.567 \pm 1.063 \mu \mathrm{g} / \mathrm{mL}\right)$ (Figure 5B; Supporting information, Table S2), indicating that nanoparticles could co-deliver AZD9291 and DOX into tumor cells and release both of them under high GSH conditions to achieve better anti-tumor effect than monotherapy. Cytotoxicity was produced and the nanoparticle system retained the cytotoxic effect of AZD9291 on NSCLC cells.

Taken together, these above results indicated that T7DSNPs/9291 could not only combine cytotoxicity of AZD9291 with DOX to cancer cells, but could also reduce the toxicity of therapeutic drugs to cerebral capillary endothelial cells.

\section{In vivo Real-Time Fluorescence Imaging}

In order to evaluate the in vivo distribution and BBB penetration ability of nanocarrier, Dir labeled PEG-DSNPs/9291 and T7-DSNPs/9291 were intravenously injected to intracranial PC-9 tumor nude mice to investigate the fluorescence distribution, respectively. From Figure 6A, we can see that the fluorescence intensity of the T7-DSNPs/9291 group is obviously higher at any time point than the PEG-DSNPs /9291 group in the intracranial PC-9 tumor-bearing brain. At 12 and 24 h post-administration, PEG-DSNPs/9291 was rarely detected in the brain, while T7-DSNPs/9291 showed strong intensity in the brain. Twenty-four hours after injection, the distribution of nanoparticles in the mouse brain tissue section was qualitatively observed using a laser confocal microscope (Figure 6B). The red fluorescence intensity in the brain of the T7-DSNPs/9291 group was significantly stronger than that of the PEG-DSNPs/9291 group. The ex vivo fluorescence of the brain also confirmed the accumulation of the T7-DSNPs/9291 group was much more than the
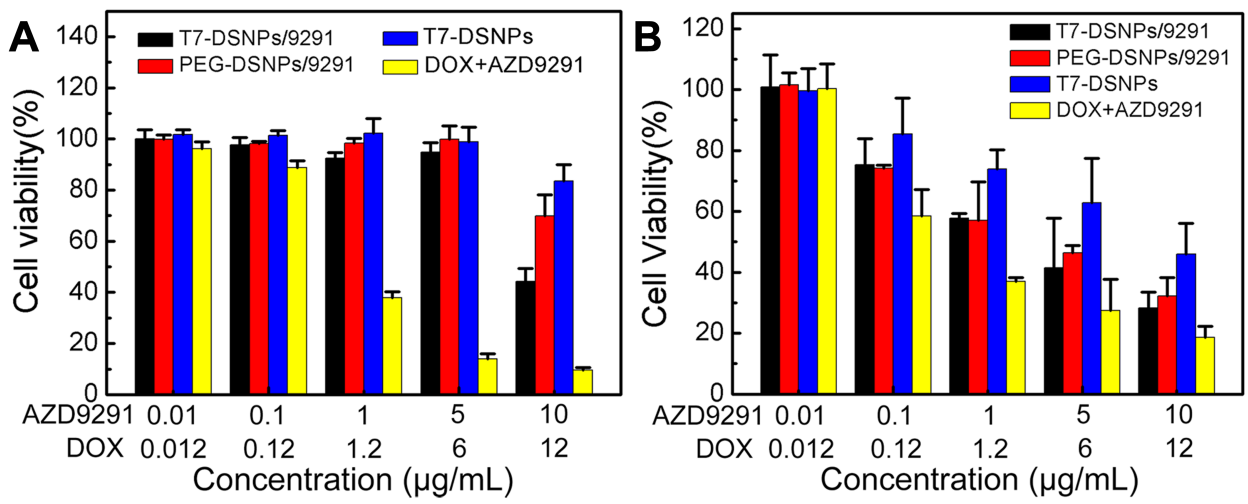

Figure 5 Cytotoxicity of AZD929I + DOX, T7-DSNPs, PEG-DSNPs/929I, and T7-DSNPs/929I in BCEC cells $(\mathrm{n}=6)(\mathbf{A})$ or PC-9 cells for $48 \mathrm{~h}(\mathrm{n}=6)(\mathbf{B})$. 

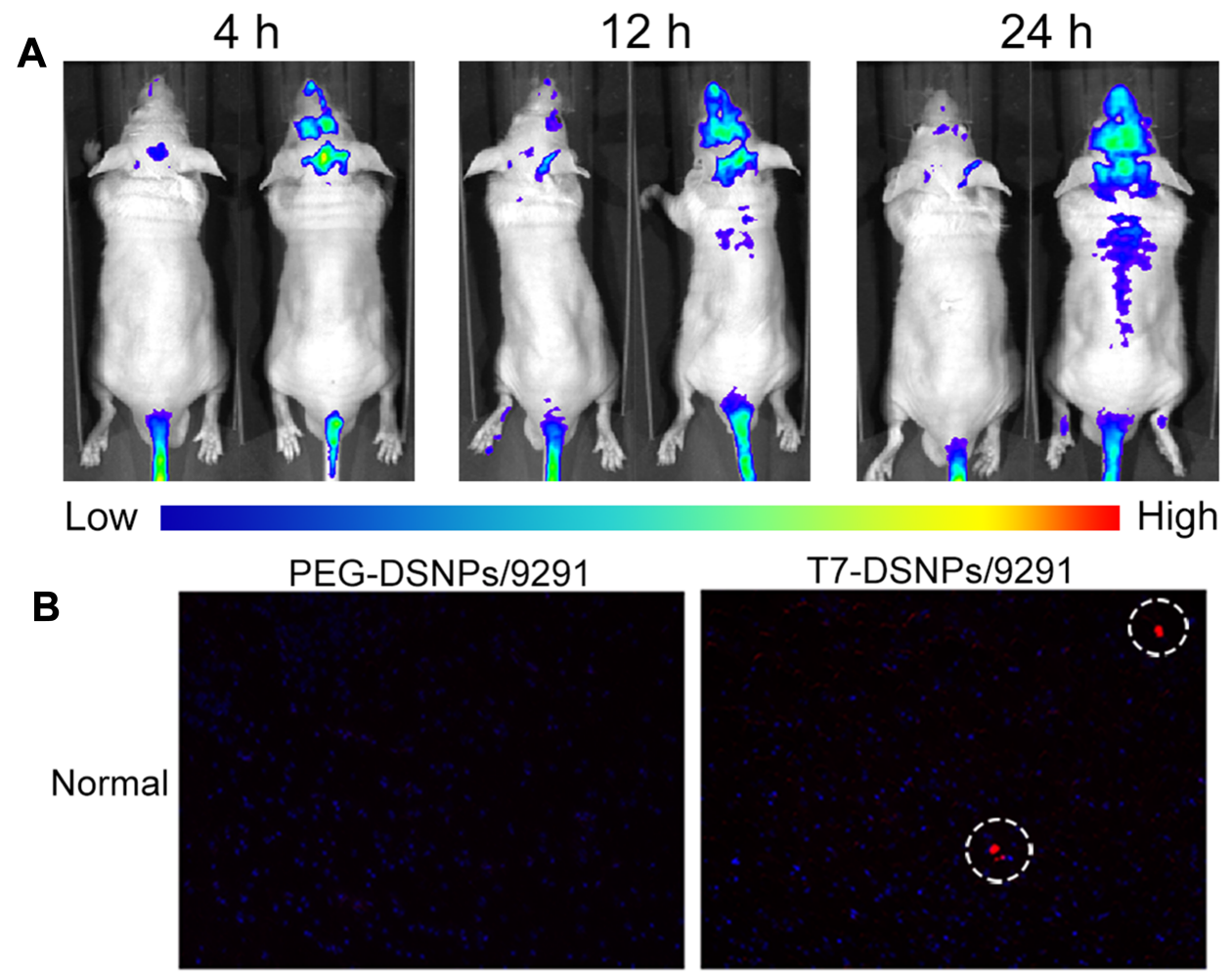

\section{High}

B
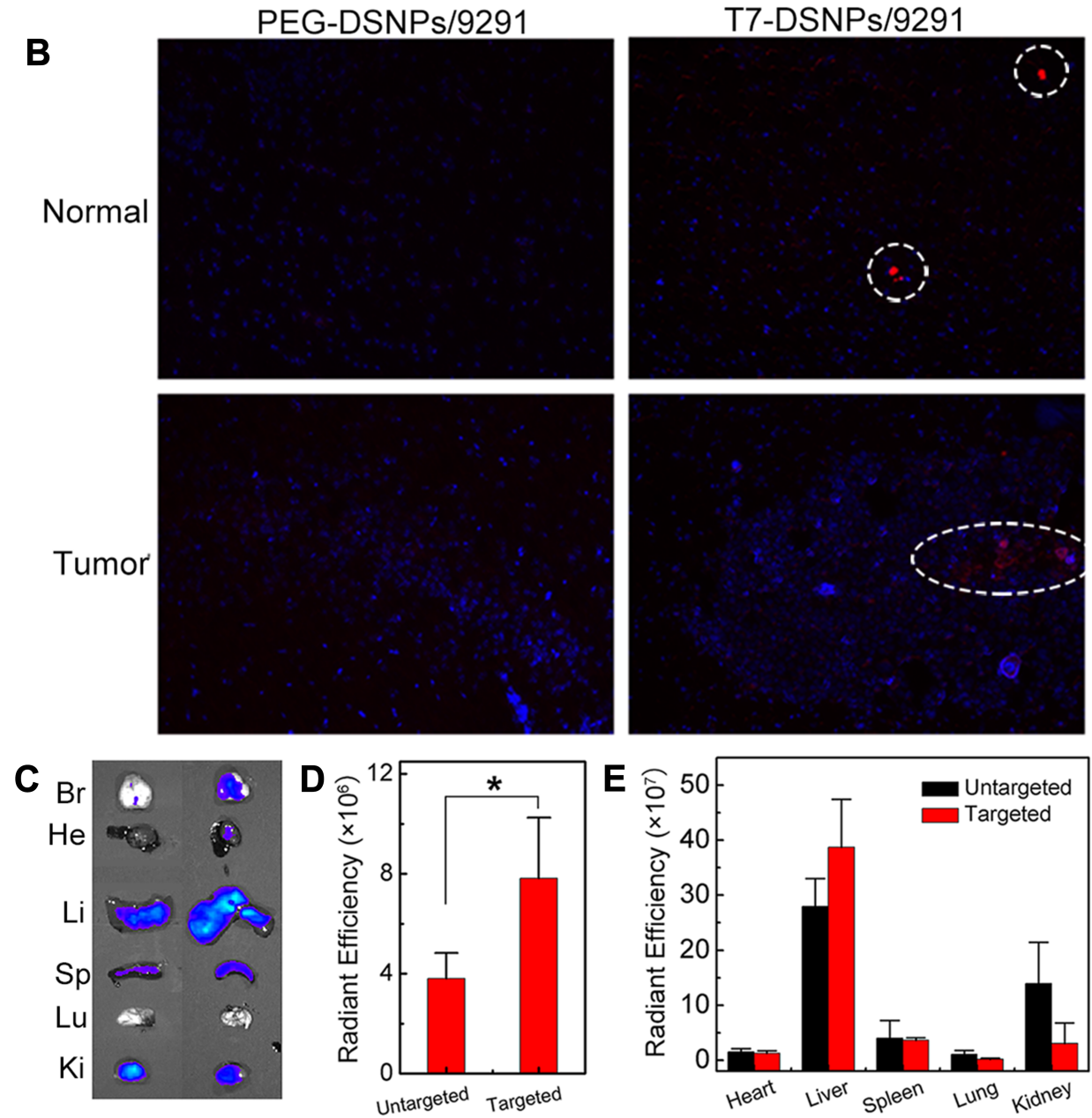

Figure 6 In vivo distribution of T7-DSNPs/929I. In vivo real-time fluorescence distribution of intracranial PC-9 tumor mice administrated with Dir labeled PEG-DSNPs 1929I (left, untargeted) and T7-DSNPs/929I (right, targeted) at 4, 12, 24 h post-injection (A). The brain section distribution of PEG-DSNPs/929I and T7-DSNPs/929I of intracranial PC-9 tumor nude mice after 24 hours of administration. Scale is $200 \times$. Blue, DAPI; red, Dir; Bar: $400 \mu \mathrm{m}$ (B). Ex vivo fluorescence distribution in main organs 24 h post-administration with Dir labeled T7-DSNPs/929I (right) and PEG-DSNPs/929I (left) (C). The semiquantitative radiant analysis of brains, *P < 0.05 (D) and organs (E). Abbreviations: Br, brain; He, heart; Li, liver; Sp, spleen; Lu, lung; Ki, kidney.

PEG-DSNPs/9291 group in the brain (Figure 6C). Similarly, the corresponding semi-quantitative results for the brain showed that the tumor fluorescence of the T7-DSNPs/9291 group was 2.05 times higher than the PEG-DSNPs/9291 group (Figure 6D). These results indicated that T7 peptide modification could significantly increase the BBB transport of nanocarriers by TfR-mediated transcytosis. Therefore, the AZD9291 and DOX co-delivery nanoparticles could be 

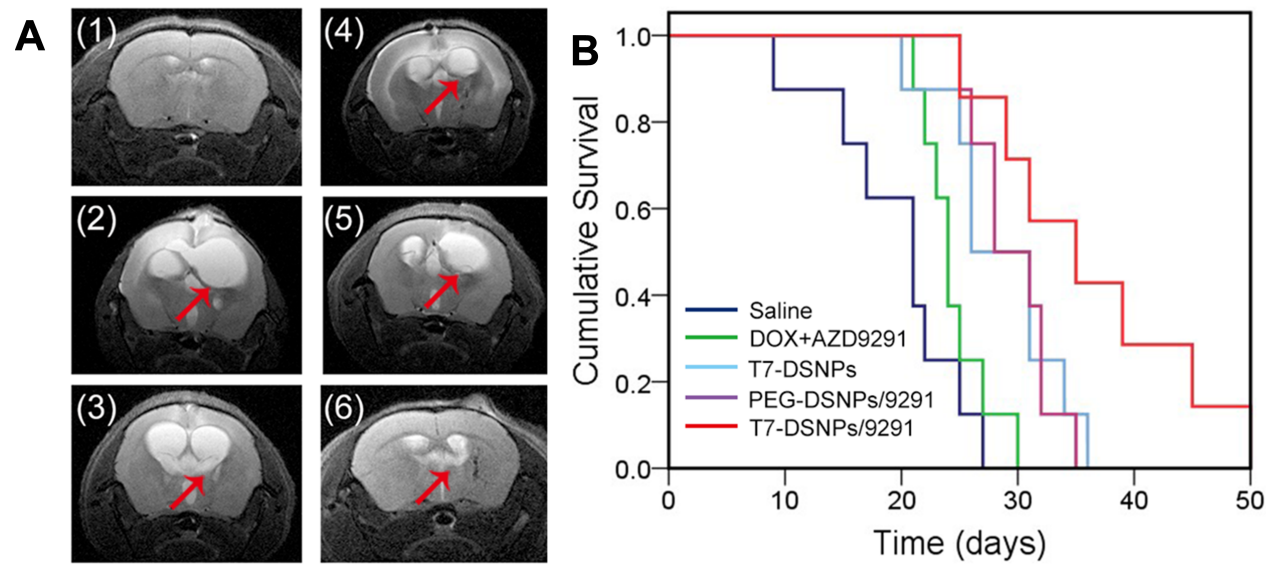

Figure 7 In vivo antitumor efficiency of T7-DSNPs/929I. Representative brain MRI images of the normal mice (I) and intracranial PC-9 orthotopic transplanted mice administrated with saline (2), AZD929I+ DOX (3), T7-DSNPs (4), PEG-DSNPs/929I (5), and T7-DSNPs/929I (6) (A). Kaplan-Meier survival curve of intracranial PC-9 tumor mice administrated with various formulations every three days 4 times after PC-9 implantation at a dose of $5 \mathrm{mg} / \mathrm{kg}$ AZD929/and $6 \mathrm{mg} / \mathrm{kg}$ DOX $(\mathrm{n}=8)(\mathbf{B})$.

utilized as a brain-targeted drug carrier for the treatment of brain metastases from NSCLC.

However, the in vivo real-time fluorescence distribution also indicated that the T7-DSNPs/9291 and the PEGDSNPs/9291 were mainly accumulated into the liver, which might be due to the nonspecific uptake of nanoparticles by the reticuloendothelial system (Figure 6E).

\section{In vivo Anti-Brain Metastasis From NSCLC Efficacy}

Brain MRI showed that the size of NSCLC brain metastasis tumor of mice treated with T7-DSNPs/9291 nanoparticles was significantly smaller than the other four groups at 21 days, and T7-DSNPs/9291 significantly inhibited the metastasis of NSCLC to the left side of the brain (Figure 7A). As displayed in Figure 7B, the median survival time of intracranial PC-9 tumor-bearing mice treated with T7-DSNPs/9291 was 35 days, which was the longest compared with those groups administrated with saline (21 days), free drug (24 days), T7-DSNPs (26 days) and PEG-DSNPs/9291 (28 days) (Supporting infor mation, Table S3). At the same time, the result showed that the antitumor efficiency of the nanocarrier was better than that of combined free drugs. What is more, T7DSNPs/9291 showed a better therapeutic effect than that of PEG-DSNPs/9291 since the modification of the T7 peptide improved the BBB penetrability of the nanocarrier. And the antitumor efficiency of T7-DSNPs/9291 was also better than T7-DSNPs due to the combination of DOX and AZD9291. These results indicated that the AZD9291 and DOX co-delivery nanoparticles showed a great enhancement in the efficacy against brain metastases from NSCLC compared with a single administration, which could provide a potential drug co-delivery strategy for the treatment of brain metastases from NSCLC.

\section{Conclusions}

In this study, we have constructed a GSH-responsive doxorubicin prodrug self-assembled brain-targeted nanocarrier with satisfactory AZD9291 loading capacity to deliver AZD9291 and DOX for the combined therapy of brain metastases from NSCLC. In vitro release assays explained that T7-DSNPs/9291 GSH-triggered release of AZD9291 and DOX. The cellular study indicated that T7-DSNPs /9291 could significantly enhance the uptake and penetration across BBB, and improve the anti-tumor effect on PC-9 cells while minimizing the toxicity of drug to normal cells. What is more, in vivo experiments demonstrated that T7-DSNPs /9291 significantly prolong the survival of mice. These results indicated that the T7-DSNPs/9291 was a potential brain targeted drug carrier allowing both drugs to work concurrently at the corresponding location for the combination therapy of brain metastases from NSCLC.

\section{Ethics Approval and Informed Consent}

The animal studies were approved by the ethics committee of Nanjing Medical University and the animal use protocol title approval by the institutional animal care and use committee is anti-tumor drug carrier research (IACUC1,904,007). All the animal procedures in the current study were performed in accordance with the Guidelines 
for Care and Use of Laboratory Animals of Nanjing Medical University.

\section{Acknowledgments}

This work was supported by the National Natural Science Foundation of China (81973255), 2017 Six Talent Peaks Project of Jiangsu Province (SWYY-051), Qin Lan Project of Jiangsu Province, Science and Technology Development Foundation of Nanjing Medical University (2017NJMU216), Science and Technology Plan Project of Jiangxi Province Education Department (GJJ170736) and First Class Discipline Construction Project of Jiangxi Province (JXSYLXK-ZHYAO007).

\section{Disclosure}

The authors report no conflicts of interest in this work.

\section{References}

1. Siegel RL, Miller KD, Jemal A. Cancer statistics, 2018. CA Cancer J Clin. 2018;68(1):7-30. doi:10.3322/caac.21442

2. Grinberg-Rashi H, Ofek E, Perelman M, et al. The expression of three genes in primary non-small cell lung cancer is associated with metastatic spread to the brain. Clin Cancer Res. 2009;15 (5):1755-1761. doi:10.1158/1078-0432.CCR-08-2124

3. Khuntia D, Brown P, Li J, Mehta MP. Whole-brain radiotherapy in the management of brain metastasis. J Clin Oncol. 2006;24 (8):1295-1304. doi:10.1200/JCO.2005.04.6185

4. Soffietti R, Kocher M, Abacioglu UM, et al. A European Organisation for Research and Treatment of Cancer Phase III trial of adjuvant whole-brain radiotherapy versus observation in patients with one to three brain metastases from solid tumors after surgical resection or radiosurgery: quality-of-life results. $J$ Clin Oncol. 2013;31(1):65-72. doi:10.1200/JCO.2011.41.0639

5. Li J, Brown PD. The diminishing role of whole-brain radiation therapy in the treatment of brain metastases. JAMA Oncol. 2017;3 (8):1023-1024. doi:10.1001/jamaoncol.2016.5411

6. D'Antonio C, Passaro A, Gori B, et al. Bone and brain metastasis in lung cancer: recent advances in therapeutic strategies. Ther Adv Med Oncol. 2014;6(3):101-114. doi:10.1177/1758834014521110

7. Cross DA, Ashton SE, Ghiorghiu S, et al. AZD9291, an irreversible EGFR TKI, overcomes T790M-mediated resistance to EGFR inhibitors in lung cancer. Cancer Discov. 2014;4(9):1046-1061. doi:10.1158/2159-8290.CD-14-0337

8. Wu YL, Ahn MJ, Garassino MC, et al. CNS efficacy of osimertinib in patients with T790M-positive advanced non-small-cell lung cancer: data from a randomized phase III trial (AURA3). J Clin Oncol. 2018;36(26):2702-2709. doi:10.1200/JCO.2018.77.9363

9. Mok TS, Wu YL, Ahn MJ, et al. Osimertinib or platinum-pemetrexed in EGFR T790M-positive lung cancer. $N$ Engl J Med. 2017;376 (7):629-640. doi:10.1056/NEJMoa1612674

10. Reungwetwattana T, Nakagawa K, Cho BC, et al. CNS response to osimertinib versus standard epidermal growth factor receptor tyrosine kinase inhibitors in patients with untreated EGFR-mutated advanced non-small-cell lung cancer. J Clin Oncol. 2018;36(33):3290-3297. doi:10.1200/JCO.2018.78.3118

11. Elmeliegy MA, Carcaboso AM, Tagen M, Bai F, Stewart CF. Role of ATP-binding cassette and solute carrier transporters in erlotinib CNS penetration and intracellular accumulation. Clin Cancer Res. 2011;17 (1):89-99. doi:10.1158/1078-0432.CCR-10-1934
12. Burel-Vandenbos F, Ambrosetti D, Coutts M, Pedeutour F. EGFR mutation status in brain metastases of non-small cell lung carcinoma. J Neurooncol. 2013;111(1):1-10. doi:10.1007/s11060-012-0990-5

13. Berger LA, Riesenberg H, Bokemeyer C, Atanackovic D. CNS metastases in non-small-cell lung cancer: current role of EGFR-TKI therapy and future perspectives. Lung Cancer. 2013;80(3):242-248. doi:10.1016/j.lungcan.2013.02.004

14. Robert N, Leyland-Jones B, Asmar L, et al. Randomized phase III study of trastuzumab, paclitaxel, and carboplatin compared with trastuzumab and paclitaxel in women with HER-2-overexpressing metastatic breast cancer. J Clin Oncol. 2006;24(18):2786-2792. doi:10.1200/JCO.2005.04.1764

15. Wang Y, Liu S, Wei X, et al. Non-small cell lung cancer leptomeningeal metastases treated with intrathecal therapy plus osimertinib and temozolomide and whole-brain radiation therapy: a case report. Onco Targets Ther. 2018;11:4733-4738. doi:10.2147/OTT. S164968

16. Tacar O, Sriamornsak P, Dass CR. Doxorubicin: an update on anticancer molecular action, toxicity and novel drug delivery systems. J Pharm Pharmacol. 2013;65(2):157-170. doi:10.1111/j.2042-7158. 2012.01567.x

17. Taratula O, Kuzmov A, Shah M, Garbuzenko OB, Minko T. Nanostructured lipid carriers as multifunctional nanomedicine platform for pulmonary co-delivery of anticancer drugs and siRNA. J Control Release. 2013;171(3):349-357. doi:10.1016/j.jconrel.20 13.04.018

18. Xu X, Ho W, Zhang X, Bertrand N, Farokhzad O. Cancer nanomedicine: from targeted delivery to combination therapy. Trends $\mathrm{Mol}$ Med. 2015;21(4):223-232. doi:10.1016/j.molmed.2015.01.001

19. Huang W, Chen L, Kang L, et al. Nanomedicine-based combination anticancer therapy between nucleic acids and small-molecular drugs. Adv Drug Deliv Rev. 2017;115:82-97. doi:10.1016/j.addr.2017. 06.004

20. Xiao B, Ma L, Merlin D. Nanoparticle-mediated co-delivery of chemotherapeutic agent and siRNA for combination cancer therapy. Expert Opin Drug Deliv. 2017;14(1):65-73. doi:10.1080/174252 47.2016.1205583

21. Meng F, Wang J, Ping Q, Yeo Y. Camouflaging nanoparticles for ratiometric delivery of therapeutic combinations. Nano Lett. 2019;19 (3):1479-1487. doi:10.1021/acs.nanolett.8b04017

22. Saito G, Swanson JA, Lee KD. Drug delivery strategy utilizing conjugation via reversible disulfide linkages: role and site of cellular reducing activities. Adv Drug Deliv Rev. 2003;55(2):199-215. doi:10.1016/S0169-409X(02)00179-5

23. Brulisauer L, Gauthier MA, Leroux JC. Disulfide-containing parenteral delivery systems and their redox-biological fate. $J$ Control Release. 2014;195:147-154. doi:10.1016/j.jconrel.2014.06.012

24. Zhang Z, Cheng W, Pan Y, Jia L. An anticancer agent-loaded PLGA nanomedicine with glutathione-response and targeted delivery for the treatment of lung cancer. $J$ Mat Chem B. 2020;8(4):655-665. doi:10.1039/C9TB02284H

25. Wang Y, Liu D, Zheng Q, et al. Disulfide bond bridge insertion turns hydrophobic anticancer prodrugs into self-assembled nanomedicines. Nano Lett. 2014;14(10):5577-5583. doi:10.1021/n1502044x

26. Chi Y, Yin X, Sun K, et al. Redox-sensitive and hyaluronic acid functionalized liposomes for cytoplasmic drug delivery to osteosarcoma in animal models. J Control Release. 2017;261:113-125. doi:10.1016/j.jconrel.2017.06.027

27. Jiang Y, Wang X, Liu X, et al. Enhanced antiglioma efficacy of ultrahigh loading capacity paclitaxel prodrug conjugate self-assembled targeted nanoparticles. ACS Appl Mater Interfaces. 2017;9(1):211-217. doi:10.1021/acsami.6b13805

28. Lee SY, Kim S, Tyler JY, Park K, Cheng JX. Blood-stable, tumor-adaptable disulfide bonded mPEG-(Cys)4-PDLLA micelles for chemotherapy. Biomaterials. 2013;34(2):552-561. doi:10.1016/j. biomaterials.2012.09.065 
29. Lu L, Li B, Lin C, et al. Redox-responsive amphiphilic camptothecin prodrug nanoparticles for targeted liver tumor therapy. $J$ Mat Chem B. 2020;8(17):3918-3928.

30. Zhao Y, Jiang Y, Lv W, et al. Dual targeted nanocarrier for brain ischemic stroke treatment. $J$ Control Release. 2016;233:64-71. doi:10.1016/j.jconrel.2016.04.038

31. Wang Z, Zhao Y, Jiang Y, et al. Enhanced anti-ischemic stroke of ZL006 by T7-conjugated PEGylated liposomes drug delivery system. Sci Rep. 2015;5(1):12651. doi:10.1038/srep12651

32. Leng X, Ma J, Liu Y, et al. Mechanism of piR-DQ590027/MIR17HG regulating the permeability of glioma conditioned normal BBB. $J$ Exp Clin Cancer Res. 2018;37(1):246. doi:10.1186/s13046-018-0886-0
33. Niessner H, Schmitz J, Tabatabai G, et al. PI3K pathway inhibition achieves potent antitumor activity in melanoma brain metastases in vitro and in vivo. Clin Cancer Res. 2016;22(23):5818-5828. doi:10.1158/1078-0432.CCR-16-0064

34. Chan KH, Lee WH, Ni M, Loo Y, Hauser CAE. C-Terminal residue of ultrashort peptides impacts on molecular self-assembly, hydrogelation, and interaction with small-molecule drugs. Sci Rep. 2018;8:17127. doi:10.1038/s41598-018-35431-2

\section{Publish your work in this journal}

The International Journal of Nanomedicine is an international, peerreviewed journal focusing on the application of nanotechnology in diagnostics, therapeutics, and drug delivery systems throughout the biomedical field. This journal is indexed on PubMed Central, MedLine, CAS, SciSearch ${ }^{\mathbb{B}}$, Current Contents ${ }^{\mathbb{B}} /$ Clinical Medicine, $^{2}$
Journal Citation Reports/Science Edition, EMBase, Scopus and the Elsevier Bibliographic databases. The manuscript management system is completely online and includes a very quick and fair peer-review system, which is all easy to use. Visit http://www.dovepress.com/ testimonials.php to read real quotes from published authors. 